\title{
Ecology of Hydrocorals-An Overview of the Relevance of New Conservation Strategies to Counteract Coral Reef Threats
}

\author{
Víctor Hugo Hernández Elizárraga* \\ Facultad de Química, Universidad Autónoma de Querétaro, Mexico
}

Submission: December 21, 2020; Published: January 29, 2021

Corresponding author: Víctor Hugo Hernández Elizárraga, Posgrado en Ciencias Químico Biológicas, Facultad de Química, Universidad Autónoma de Querétaro, Querétaro, Mexico

\begin{abstract}
Hydrocorals (Phylum Cnidaria, Class Hydrozoa, Genus Millepora) are recognized as the second most important reef-builders. However, despite their ecological and toxicological relevance, very little scientific research has been conducted on these organisms. Overall, the current landscape of reef-building organisms in the Anthropocene result in discouraging due to climate change. Up to date, the scientific community has proposed some strategies to ameliorate the damage induced to marine species by climate change. However, these efforts have been poorly effective and only considered scleractinian corals (Class Anthozoa), while Hydrocorals remain neglected.
\end{abstract}

Keywords: Hydrocorals; Fire corals; Millepora; Coral bleaching; Thermal stress

\section{Opinion}

Hydrocorals (Phylum Cnidaria, Class Hydrozoa, Genus Millepora) are recognized as the second most important reefbuilders in the world just behind scleractinian corals (Phylum Cnidaria, Class Anthozoa). These organisms play a critical role in Earth's economy since they can provide goods and services with great economic benefits such as fishing and tourism. On the other hand, one of the most remarkable features of the reefforming organisms is their ability to producing calcium carbonate skeletons. Coral skeletons form tridimensional structures or "submarine rainforests" that enable the settlement and shelter of many marine species. Besides, coral reefs can prosper in oligotrophic environments, where nutrients are very scarce, due to the mutualistic symbiosis with photosynthetic algae of the Symbiodiniaceae family. But also, these reef-budding cnidarians can establish a complex relationship with associated microorganisms. Due to these reasons, reef-forming cnidarians are considered as holobionts, which display interesting aspects that remain to be discovered.

Being part of the most venomous Phylum on the globe, Hydrocorals display a high toxic capacity, they are commonly known as stinging "fire corals". The "fire corals" venom is stored in the nematocyst, cellular organelles specialized in prey capture. It is very common that snorkelers or divers get injured by these shallow-water organisms. A hydrocoral sting can induce burn, rashes, blisters, itch, and even kidney damage and pulmonary edema. Up to date, there is no treatment for the lesions induced by "fire corals". However, despite their ecological and toxicological relevance, very few scientific research has been conducted on these organisms. For example, little is known about the chemical structure of Millepora toxins. Millepora complanata, M. alcicornis, M. platyphylla and M. squarrosa are some representative species from this group of venomous marine metazoans, which are ubiquitous constituents of reef barriers. Further, platelike hydrocorals such as $M$. complanata can offer protection to coast preventing erosion by absorbing the swell during storms and hurricanes, these aspects make them essential for coastal protection.

Overall, the current landscape of reef-building organisms in the Anthropocene result in discouraging due to climate change. Some of the main threats that coral and hydrocorals are facing including pollution, ocean acidification, high UV radiation, and thermal stress. Considering that due to global warming the average temperature of our planet has increased approximately 1.5 degrees 
Celsius , thermal stress represent principal factor which could lead to corals extinction. Particularly, human-induced stressors like thermal stress, have elicited massive mortality at different depths and locations around the world. The principal issue regarding coral survival is the bleaching phenomenon that has become prevalent due to warm waves such as "El Niño Southern Oscillation" (ENSO). During coral bleaching, a breakdown between the elements that constitute the holobiont occurs, where Symbiodiniaceae algae are the most affected. Coral bleaching decreases considerably the growth, reproduction, and resilience of reef-builders organisms. Up to date, the scientific community has proposed some strategies to ameliorate the damage produced by thermal stress. Some tools addressing to counteract coral bleaching including reforestation, symbiont switching, chimerism, ecological engineering, naturebased solutions, among others. However, these efforts have been poorly effective and only considered scleractinian corals (Class Anthozoa), while Hydrocorals remain neglected. Near 25\% of marine species depends on coral reefs, if corals extinguish, this will impact severely a quarter of marine life.

At this stage, humanity must slow down or compensate for its accelerated growth and provide corals clean seawater and stop the rise in ocean temperatures. The truth is that the incidence and severity of global scale coral-mortality events due to thermal stress have augmented considerably. Thus, the understanding of the effects of thermal stress, or climate change in general, involved in survival processes of marine organisms like hydrocorals, will allow to designing strategies whose purpose will be to prevent or reverse coral bleaching. In addition to the enormous ecological value of reef-forming organisms, corals and hydrocorals are crucial for areas such as biotechnology and pharmacology, because they represent priceless models for the development of new research tools and drugs. It is known that Hydrocorals and many marine organisms are very susceptible to thermal stress due to global warming. Forasmuch as the development of novel strategies aimed at counteracting the impact of climate change on valuable species is urgently needed. By contrast, if the academic and non-academic communities do not act, the invaluable goods and services offered by these organisms could be lost. Which will restrict future generations to enjoy and explore the benefits that "fire corals" can provide. 